\title{
Cost effectiveness of personalized treatment in women with early breast cancer: the application of OncotypeDX and Adjuvant! Online to guide adjuvant chemotherapy in Austria
}

\author{
B. Jahnn ${ }^{1,2}$, U. Rochau ${ }^{1,2}$, C. Kurzthaler ${ }^{1,2}$, M. Hubalek ${ }^{3}$, R. Miksad $^{4}$, G. Sroczynski ${ }^{1,2}$, M. Paulden ${ }^{5,6}$,
} M. Kluibenschädl ${ }^{1,2}, M . K^{\prime} r a h n^{5}$ and U. Siebert ${ }^{1,2,7,8^{*}}$

\begin{abstract}
A Breast Cancer Outcomes model was developed at the ONCOTYROL research center to evaluate personalized testtreatment strategies in Austria. The goal was to evaluate the cost-effectiveness of a new 21-gene assay (ODX) when used in conjunction with the Adjuvant! Online (AO) decision aid to support personalized decisions about use of adjuvant chemotherapy in early-stage breast cancer patients in Austria. We applied a validated discrete-event-simulation model to a hypothetical cohort of 50 years old women over a lifetime horizon. The test-treatment strategies of interest were defined using three-letter acronyms. The first (second, third) letter indicates whether patients with a low (intermediate, high) risk according to AO were tested using ODX ( $Y$ yes, $N$ no). The main outcomes were life-years gained, quality-adjusted life-years (QALYs), costs and cost effectiveness. Robustness of the results was tested in sensitivity analyses. Results were compared to a Canadian analysis conducted by the Toronto Health Economics and Technology Assessment Collaborative (THETA). Five of eight strategies were dominated (i.e., more costly and less effective: NNY, NYN, YNN, YNY, YYN). The base-case analysis shows that YYY (ODX provided to all patients) is the most effective strategy and is cost effective with an incremental cost-effectiveness ratio of 15,700 EUR per QALY gained. These results are sensitive to changes in the probabilities of distant recurrence, age and costs of chemotherapy. The results of the base-case analysis were comparable to the THETA results. Based on our analyses, using ODX in addition to AO is effective and cost effective in all women in Austria. The development of future genetic tests may require alternative or additional test-treatment strategies to be evaluated.
\end{abstract}

Keywords: 21-Gene assay, Genetic testing, Adjuvant! Online, Cost effectiveness, Decision-analytic model, Discrete event simulation

\section{Background}

Breast cancer accounts for $28 \%$ of all malignancies in Austrian women (Statistik Austria 2012). Based on

\footnotetext{
*Correspondence: uwe.siebert@umit.at

${ }^{1}$ Institute of Public Health, Medical Decision Making and Health Technology Assessment, Department of Public Health, Health Services Research and Health Technology Assessment, UMIT - University for Health Sciences, Medical Informatics and Technology, Eduard Wallnoefer Center 1, A-6060 Hall i.T, Austria

Full list of author information is available at the end of the article
}

current projections, seven of every 100 Austrian girls born in 2009 will develop breast cancer by the age of 75 (Statistik Austria 2012). Aside from a small percentage of familial breast cancer syndromes, risk factors include age, early menarche, late menopause, and obesity [Arbeitsgemeinschaft für gynäkologische Onkologie (AGO) der Österreichischen Gesellschaft für Gynäkologie und Geburtshilfe (OEGGG) 2012]. Currently, various treatment strategies are available

\section{黛 Springer}

(c) 2015 Jahn et al. This article is distributed under the terms of the Creative Commons Attribution 4.0 International License (http://creativecommons.org/licenses/by/4.0/), which permits unrestricted use, distribution, and reproduction in any medium, provided you give appropriate credit to the original author(s) and the source, provide a link to the Creative Commons license, and indicate if changes were made. 
[Leitlinienprogramm Onkologie (OL) 2012]. When possible, surgical resection offers a potential cure. Adjuvant treatment after surgery with radiation and/ or systemic therapy (including chemotherapy and/or hormonal therapy) depends on the surgical approach, stage of the disease, hormone receptor status [e.g., estrogen receptor (ER) and progesterone receptor (PR) status], postmenopausal status, HER2/neu status, pathologic findings and co-morbidities. In part due to the length of treatment and potential severe and lethal side effects, the use of adjuvant chemotherapy has been heavily investigated in terms of its impact on risk of recurrence and overall survival.

In particular for women with lymph node negative, ER positive early-stage breast cancer, the decision about whether to treat with adjuvant chemotherapy is complex and uncertain. Adjuvant chemotherapy can be beneficial for women at higher risk of a distant recurrence, but can cause more harm for low risk patients. There are several prognostic tests available that can identify women who are most likely to benefit from adjuvant therapy. For example, Adjuvant! Online (AO) is a free, web-based decision aid designed to help patients and clinicians understand individual risk, the impact of systemic therapy. AO estimates breast cancer-specific mortality and recurrence after surgery for various chemotherapy and hormonal treatment options based on the patient's age, comorbidities, estrogen receptor status, tumor size, tumor grade and number of positive lymph nodes (Adjuvant! Online Inc 2012). MammaPrint ${ }^{\circledR}$ and OncotypeDX ${ }^{\circledR}$ are gene expression assays that quantify the risk of distant disease recurrence (Agency for Healthcare Research and Quality 2012).

We evaluated the benefit-harm balance and cost effectiveness of the 21-gene assay OncotypeDX ${ }^{\circledR}$ [Genomic Health Inc., Redwood City, CA, USA (ODX)] because it was considered potentially useful for adjuvant chemotherapy decision-making by $84 \%$ of the experts at the 2011 St. Gallen Consensus Conference (Gnant et al. 2011). In 2013, the European Society for Oncology (ESMO) concurred that in cases of uncertainty regarding adjuvant chemotherapy (after consideration of other tests), gene expression, assays such as MammaPrint ${ }^{\circledR}$ or OncotypeDX ${ }^{\circledR}$ may be used. The analytical and clinical test validity of this assay convinced the majority of the IMPACT 2012 Working Group members in its clinical use (Azim et al. 2013). However, the 21-gene assay is not currently offered in Austria.

Several studies have evaluated the impact of ODX on treatment decisions as compared to common clinical and pathological criteria such as the National Comprehensive Cancer Network (NCCN) guidelines, the NSABP B-20 study or Adjuvant! Online (AO) (Adjuvant! Online Inc 2012). Most studies have identified an impact on decisions and changes in use of chemotherapy in up to $50 \%$ of all patients (Asad et al. 2008; Henry et al. 2009; Klang et al. 2010; Lo et al. 2010; Oratz et al. 2007, 2011). For the most part, recommendations have changed from chemotherapy plus hormonal therapy to hormonal therapy alone in patients with ER-positive and LN-negative breast cancer (Henry et al. 2009; Lo et al. 2010; Liang et al. 2007). In these patients, chemotherapy and its associated adverse events may be avoided. In addition, increased confidence among medical oncologists and decreased patient anxiety were reported with the use of ODX (Lo et al. 2010) .

The cost effectiveness of using ODX has been evaluated in several studies (Klang et al. 2010; Hall et al. 2012; Kondo et al. 2008, 2011; Lyman et al. 2007; Paulden et al. 2013; Reed et al. 2009; Tsoi et al. 2010; Ward et al. 2013; Yang et al. 2012; Hornberger et al. 2005). However, physicians may want to consider clinicopathological factors (like in AO) along with genomic test results, especially when the risk classification with $\mathrm{AO}$ is validated and free of charge. We found only two studies that considered the combination of both tests (Paulden et al. 2013; Reed et al. 2009). These studies did not cover Austrian treatment patterns or costs.

Therefore, the aim of our study was to evaluate the effectiveness and the cost effectiveness of ODX when it is used or not used in conjunction with the AO score to guide decisions about use of adjuvant chemotherapy women diagnosed with ER and/or PR positive, HER-2/ neu negative, and lymph node negative breast cancer in Austria.

\section{Methods \\ Model design and assumptions}

We applied a previously published and validated decision-analytic model to estimate life years (LY) and quality-adjusted life years (QALY) (Jahn et al. 2012a, b) (Jahn et al. 2015). In addition, we also estimated the number of adverse drug events due to chemotherapy, number of patients with a distant recurrence, number of patients who die from a distant recurrence, long term costs as well as incremental cost-effectiveness ratios (ICER) for each of the eight strategies being evaluated (see next section). In the base-case analysis, a hypothetical cohort of 50-year old women diagnosed with ER and/or PR positive, HER-2/neu negative, and lymph node negative breast cancer was evaluated over their remaining lifetime. The societal 
perspective in the Austrian health care context was adopted. Only direct costs were considered to avoid double counting (Gold et al. 1996). For our Oncotyrol model, we applied a discrete event simulation model (DES) approach using ARENA (version 13.90.00000, Rockwell Automation). Our choice was guided by the ISPOR-SMDM guidelines that take into account that individual patient pathways are determined by multiple characteristics and test results, while considering time-dependent functional relationships, and recording of individual patient pathways (Karnon et al. 2012; Caro et al. 2012).

\section{Model structures}

In this individual-level computer simulation, hypothetical patients were projected over a lifelong analytic time horizon after they had received surgery. The model included 4 parts: first, patients were assigned their characteristics and test results (Module 1-risk classification); second, chemotherapy was provided if necessary (Module 2-chemotherapy); third, the time patients remained free from a distant recurrence was considered (Module 3-recurrence free); and fourth, distant recurrence was considered (Module 4-recurrence). Finally, a statistical module provided a summary of the model outcomes (Fig. 1).

In Module 1, we evaluated eight two-test strategies to determine if chemotherapy should be given. We assumed that each patient was assigned an individualized breast cancer specific mortality (BCSM) risk based on the $\mathrm{AO}$ assessment. (Adjuvant! Online Inc 2012): 'low' (BCSM $<9 \%)$, 'intermediate' $(9 \% \leq \mathrm{BCSM}<17 \%)$ or 'high' $(\mathrm{BCSM} \geq 17 \%)$ risk (Bryant 2005). The BCSM risk assigned by AO was then used to determine if genomic testing with ODX would be pursued. Because genomic testing results in additional costs and because it is recommended that ODX may be used in conjunction with all clinical and pathologic factors in cases where the decision about use of chemotherapy is difficult (Azim et al. 2013; al 2013), we assume a sequential application (AO first, followed by ODX). ODX provides a recurrence risk score between 0 and 100, in which 'low' is considered RS < 18, 'intermediate' $18 \leq \mathrm{RS}<30$ and 'high' ( $R S \geq 30$ ) (Paik et al. 2004). The eight test-treatment strategies evaluated are described using three letters: NNN, YYY, NNY, NYN, YNN, YYN, YNY and NYY. The first letter indicates whether patients with a low risk according to AO were tested using the 21-gene assay (Y-yes; $\mathrm{N}$-no), the second and the third letters provide this information for $\mathrm{AO}$ intermediate and high risk patients, respectively (e.g., NYN means that only patients with an AO intermediate risk are tested with the 21-gene assay). Adjuvant chemotherapy was then provided according to the results of both tests (see Table 1). Patients in the model move to Module 2 (adjuvant chemotherapy) or directly to Module 3 (follow-up) as appropriate. In Module 2, chemotherapy and associated adverse drug events (neutropenia, fever, infections, pain, nausea, gastrointestinal complications) were modeled. After the administration of chemotherapy, patients were considered recurrencefree and move to Module 3. In Module 3, each patient was followed until death from other causes or distant recurrence. Within the first 3 years, patients are assumed to have quarterly visits, thereafter biannually and after 5 years, annually. The regular visits consist of a clinical examination and mammography. Within the first 5 years, all patients receive aromatase inhibitors. In the case of a distant recurrence, the associated diagnostic work-up and treatment are modeled in Module 4. At any point in time, patients may die from other causes. In addition, patients receiving chemotherapy may die from adverse events and patients with metastatic disease may die from breast cancer. Within the simulation, information on patients' remaining lifetime and quality-adjusted lifetime, adverse drug events, and costs are accumulated and final outcomes are computed at the end of their simulated lifetime.

The model structure and a comprehensive model validation process are described in more detail elsewhere (Jahn et al. 2012a, b, 2015).

\section{Model parameters \\ Clinical parameters}

Table 1 provides an overview of model parameters and sources.

The proportion of patients assigned to each of the 12 risk groups according to AO and ODX was based on a retrospective study (Paulden et al. 2013). The provision of chemotherapy within the twelve risk groups was based on a prospective study in a North American population (Lo et al. 2010) and adapted based on Austrian expert opinion (Medical University Innsbruck 2012) for the AO low risk groups. Time to recurrence for different risk classes was derived from Paulden et al. (2013). Because there are no published prospective studies that report distant recurrences conditional on 21-gene assay or AO risk, Paulden et al. (2013) used findings from a retrospective analysis based on a subset of the NSABP B-14 study (Bryant 2005) and from a subset of the NSABP B-20 study (Paik et al. 2006). 


\section{Breast cancer patients post surgery}



Fig. 1 Schematic model structure (ER/PR + : estrogen and/or progesterone receptor positive, LY: life years, QALY: quality adjusted life years, ADE: adverse drug event, Int:: intermediate, AO: Adjuvant!Online, ODX: OncotypeDX, N/A: not applied) Source: Jahn et al. (2015). Lessons Learned from a Cross-Model Validation between a Discrete Event Simulation Model and a Cohort State-Transition Model for Personalized Breast Cancer Treatment. Medical Decision Making (published ahead of print). Copyright $\odot 2015$ by Society for Medical Decision Making. Reprinted by permission of SAGE Publications, Inc.

Fatal toxicity of chemotherapy includes $0.1 \%$ of patients that will subsequently develop acute myeloid leukemia.

For patients with distant recurrence, we assumed that the probability of death due to breast cancer was identical in all patients regardless of the ER/PR status or patients' personal cancer history. Median survival for these patients was estimated as 25.8 months (Medical University Innsbruck 2012). We assumed that the time to recurrence in recurrence-free patients and the time to death in patients with recurrence was exponentially distributed.
All-cause mortality was extrapolated using life tables from Statistics Austria (2012).

\section{Cost data}

The price of the 21-gene assay was adapted from the manufacturer's suggested retail price for Austria (Jahn, Personnel email-communication with manufacturer 2012, unpublished). Direct costs for chemotherapy, cancer follow-up, and diagnosis and treatment of recurrent malignancies were based on internal calculations of the 
Table 1 Model parameter overview

\begin{tabular}{|c|c|c|}
\hline Parameters & Values & Sources \\
\hline \multicolumn{3}{|c|}{ Proportion of patients assigned to each risk group } \\
\hline Adjuvant! Online low risk & $52.99 \%$ & Paulden et al. (2013) \\
\hline 21-Gene assay low risk & $32.34 \%$ & \\
\hline 21-Gene assay int. risk & $12.57 \%$ & \\
\hline 21-Gene assay high risk & $8.08 \%$ & \\
\hline Adjuvant! Online int. risk & $18.71 \%$ & \\
\hline 21-Gene assay low risk & $8.53 \%$ & \\
\hline 21-Gene assay int. risk & $3.59 \%$ & \\
\hline 21-Gene assay high risk & $6.59 \%$ & \\
\hline Adjuvant! Online high risk & $28.29 \%$ & \\
\hline 21-Gene assay low risk & $9.73 \%$ & \\
\hline 21-Gene assay int. risk & $6.14 \%$ & \\
\hline 21-Gene assay high risk & $12.43 \%$ & \\
\hline \multicolumn{3}{|c|}{ Proportion of patients in each risk group provided adjuvant chemotherapy ${ }^{a}$} \\
\hline Adjuvant! Online low risk & $0 \%$ & Lo et al. (2010); Medical University Innsbruck (2012) \\
\hline 21-Gene assay low risk & $0 \%$ & \\
\hline 21-Gene assay int. risk & $17.62 \%$ & \\
\hline 21-Gene assay high risk & $63.44 \%$ & \\
\hline Adjuvant! Online int. risk & $55.06 \%$ & \\
\hline 21-Gene assay low risk ${ }^{a}$ & $13.73(\mathrm{~T} 1$, base case $) /(0 \% \mathrm{~T} 2, \mathrm{SA})$ & \\
\hline 21-Gene assay int. risk & $36.56 \%$ & \\
\hline 21-Gene assay high risk & $98.61 \%$ & \\
\hline Adjuvant! Online high risk & $57.57 \%$ & \\
\hline 21-Gene assay low risk & $13.72 \%$ & \\
\hline 21-Gene assay int. risk & $36.65 \%$ & \\
\hline 21-Gene assay high risk & $99.73 \%$ & \\
\hline Risk of hospital visit due to toxicity & $17.04 \%$ & Tilak Financial Department and Cost Data Report (2012) \\
\hline \multicolumn{3}{|l|}{ Cause of hospital visits due to toxicity } \\
\hline Neutropenia/fever/infections & $53.56 \%$ & Medical University Innsbruck (2012) \\
\hline Pain \& pain management & $7.51 \%$ & \\
\hline Nausea/vomiting/dehydration & $6.02 \%$ & \\
\hline Gastrointestinal tract & $5.64 \%$ & \\
\hline \multicolumn{3}{|c|}{10 year risk for distant recurrence without chemotherapy } \\
\hline Adjuvant! Online low risk & $5.39 \%$ & Paulden et al. (2013) \\
\hline 21-Gene assay low risk & $2.61 \%$ & \\
\hline 21-Gene assay int. risk & $3.84 \%$ & \\
\hline 21-Gene assay high risk & $18.91 \%$ & \\
\hline Adjuvant! Online int. risk & $20.36 \%$ & \\
\hline 21-Gene assay low risk & $4.24 \%$ & \\
\hline 21-Gene assay int. risk & $14.90 \%$ & \\
\hline 21-Gene assay high risk & $44.23 \%$ & \\
\hline Adjuvant! Online high risk & $24.12 \%$ & \\
\hline 21-Gene assay low risk & $4.24 \%$ & \\
\hline 21-Gene assay int. risk & $14.90 \%$ & \\
\hline 21-Gene assay high risk & $44.23 \%$ & \\
\hline
\end{tabular}


Table 1 continued

\begin{tabular}{|c|c|c|}
\hline Parameters & Values & Sources \\
\hline \multicolumn{3}{|l|}{10 year risk for distant recurrence with chemotherapy } \\
\hline Adjuvant! Online low risk & $5.68 \%$ & Paulden et al. (2013) \\
\hline 21-Gene assay low risk & $4.98 \%$ & \\
\hline 21-Gene assay int. risk & $5.62 \%$ & \\
\hline 21-Gene assay high risk & $8.58 \%$ & \\
\hline Adjuvant! Online int. risk & $7.35 \%$ & \\
\hline 21-Gene assay low risk & $5.79 \%$ & \\
\hline 21-Gene assay int. risk & $8.18 \%$ & \\
\hline 21-Gene assay high risk & $8.91 \%$ & \\
\hline Adjuvant! Online high risk & $7.68 \%$ & \\
\hline 21-Gene assay low risk & $5.79 \%$ & \\
\hline 21-Gene assay int. risk & $8.18 \%$ & \\
\hline 21-Gene assay high risk & $8.91 \%$ & \\
\hline Risk of mortality due to toxicity from chemotherapy & $0.1 \%$ & Medical University Innsbruck (2012) \\
\hline $\begin{array}{l}\text { Median life expectancy following distant recurrence } \\
\text { (months) }\end{array}$ & 25.8 & Medical University Innsbruck (2012) \\
\hline Risk of mortality due to other causes & Life table & Statistik Austria (2012) \\
\hline \multicolumn{3}{|l|}{ Costs (inflated to 2011 Euros) } \\
\hline 21-Gene assay & 3180 & $\begin{array}{l}\text { Jahn, Personnel email-communication with manufacturer } \\
\text { (2012, unpublished) }\end{array}$ \\
\hline \multicolumn{3}{|l|}{ Costs for chemotherapy } \\
\hline Echocardiography (one time) & 28 & Tilak Financial Department and Cost Data Report (2012) \\
\hline Chest radiography (one time) & 23 & \\
\hline Port implantation (one time) & 550 & \\
\hline Laboratory test (per cycle of chemotherapy) & 46.5 & \\
\hline Blood panel (per week for 6 months) & 3.75 & \\
\hline Human resources (per cycle of chemotherapy) & 48 & \\
\hline Hospitalization (3 days) & 620 & \\
\hline Total additional costs for chemotherapy (6 months) & $2,089.5$ & \\
\hline \multicolumn{3}{|c|}{$\begin{array}{l}\text { Total costs for FEC (Fluorouracil } 500 \mathrm{mg} / \mathrm{m}^{2} \text {, Cyclophospa- } 672.16 \\
\text { mid } 600 \mathrm{mg} / \mathrm{m}^{2} \text {, Epirubicin } 90 \mathrm{mg} / \mathrm{m}^{2} \text { ) }\end{array}$} \\
\hline Total costs for DOC (Docetaxel 75 mg/m²) & 1042.5 & \\
\hline Pegfilgrastim 6 mg & 1175.57 & \\
\hline Tropisetron-Hydrochlorid 5 mg 5 pills & 85.90 & \\
\hline $\begin{array}{l}\text { Total costs for chemotherapy (Additional costs, FEC, DOC, } \\
\text { Pegfilgrastim, Tropisetron-Hydrochlorid, } 6 \text { months) }\end{array}$ & $11,372.96$ & $\begin{array}{l}\text { Derived from Tilak Financial Department and Cost Data } \\
\text { Report (2012); Medical University Innsbruck (2012) }\end{array}$ \\
\hline \multicolumn{3}{|c|}{ Follow up costs for the first 5 years after chemo therapy (costs per month/treatment) } \\
\hline Anastozol $1 \mathrm{mg}$ & 73.7 & Tilak Financial Department and Cost Data Report (2012) \\
\hline Lertozolum 2.5 mg & 101.2 & \\
\hline Exemestanum $25 \mathrm{mg}$ & 75.87 & \\
\hline Other treatment costs for the first 5 years after chemo & 5016.8 & $\begin{array}{l}\text { Derived from Tilak Financial Department and Cost Data } \\
\text { Report (2012); Medical University Innsbruck (2012) }\end{array}$ \\
\hline Mammography & 32 & Tilak Financial Department and Cost Data Report (2012) \\
\hline Examination & 85.5 & \\
\hline Follow up costs for the first 5 years after chemo per month & 21.54 & $\begin{array}{l}\text { Derived from Tilak Financial Department and Cost Data } \\
\text { Report (2012); Medical University Innsbruck (2012) }\end{array}$ \\
\hline \multicolumn{3}{|c|}{ Follow up Costs after the first 5 years after chemo therapy (costs per month/treatment) } \\
\hline Follow up costs per month & 9.79 & $\begin{array}{l}\text { Derived from Tilak Financial Department and Cost Data } \\
\text { Report (2012); Medical University Innsbruck (2012) }\end{array}$ \\
\hline \multicolumn{3}{|l|}{ Costs of diagnosing distant recurrence } \\
\hline Total costs of diagnosis of distant recurrence & 248.5 & $\begin{array}{l}\text { Derived from Tilak Financial Department and Cost Data } \\
\text { Report (2012); Medical University Innsbruck (2012) }\end{array}$ \\
\hline
\end{tabular}


Table 1 continued

\begin{tabular}{|c|c|c|}
\hline Parameters & Values & Sources \\
\hline \multicolumn{3}{|l|}{ Costs of treating distant recurrence } \\
\hline Total costs per 25.8 months & $32,015.26$ & Tilak Financial Department and Cost Data Report (2012) \\
\hline \multicolumn{3}{|l|}{ Treatment of non-fatal chemotherapy toxicity } \\
\hline Neutropenia/fever/infections & 5231.46 & \multirow{4}{*}{$\begin{array}{l}\text { Ontario Case Costing Initiative (2011); Medical University } \\
\text { Innsbruck (2012) }\end{array}$} \\
\hline Pain management & 3270.66 & \\
\hline Nausea/vomiting/dehydration & 3173.45 & \\
\hline Gastrointestinal tract & 5169.31 & \\
\hline Treatment of fatal toxicity & 36,260 & Walter (2012) \\
\hline \multicolumn{3}{|l|}{ Utility weights } \\
\hline $\begin{array}{l}\text { First year following diagnosis (while on hormone } \\
\text { therapy) }\end{array}$ & 0.744 & Lidgren et al. (2007) \\
\hline First year following diagnosis (while on chemotherapy) & 0.620 & \\
\hline Second and following years prior to distant recurrence & 0.779 & \\
\hline Following distant recurrence & 0.685 & \\
\hline Dead & 0 & \\
\hline
\end{tabular}

a Parameter values differ for the two treatment strategies $\mathrm{T} 1$ (base case)/T2 (sensitivity analysis)

financial department of the Tyrolienne Hospital Operating Company (TILAK) (Tilak Financial Department and Cost Data Report 2012) and expert opinion from the Innsbruck Medical University (Medical University Innsbruck 2012). Drug costs were based on pharmacy hospital prices.

Costs for chemotherapy included the costs of chemotherapeutic agents, other supportive medications such as pegfilgrastim and tropisteron, hospitalization, laboratory studies, and human resources. It was assumed that all patients receive the current standard of care in terms of chemotherapeutic agents. At present, this is three cycles of FEC (5-Fluorouracil, Epirubicin, Cyclophosphamid) followed by 3 cycles of DOC (Docetaxel) (Hubalek 2010).

All patients also received an aromatase inhibitor (anastozol or lertozolum or aromasin) for 5 years. This was begun immediately in all cases except in those who received chemotherapy, in which case the aromatase inhibitor was started following the completion of adjuvant chemotherapy.

Underlying assumptions regarding the treatment of distant recurrence were derived from chart reviews by a senior gynecologist at Innsbruck Medical Hospital.

Costs associated with adverse drug events of chemotherapy were based on published data (Ontario Case Costing Initiative 2011) and adapted according to Austrian experts (Medical University Innsbruck 2012). Treatment of fatal toxicity included treatment of acute myeloid leukemia. Cost data were evaluated based on German DGHO treatment guidelines (DGHO guidelines 2013). Cost were derived from or inflated to Euros 2011. Costs and health outcomes were discounted at $5 \%$ (Walter and Zehetmayr 2006).

\section{Utility data}

Utility weights for the different breast cancer states were derived from a recent cross-sectional observational study (Lidgren et al. 2007) using the EQ-5D, a standardized, non-disease-specific instrument for describing and valuing health states (while on hormone therapy, while on chemotherapy, prior to distant recurrence, following distant recurrence).

\section{Analysis}

\section{Base-case analysis}

We estimated discounted LYs, QALYs, number of patients who experienced adverse drug events, disease recurrence, costs, and ICERs (additional costs per QALY gained). For the cost-effectiveness results, we excluded dominated strategies. These are strategies that provide fewer QALYs at a higher cost. We also excluded strategies due to extended dominance. Strategies are extendedly dominated if there is a more expensive strategy with a lower ICER. (Cantor 1994).

The cost-effectiveness results are displayed on a costeffectiveness plane. The resulting line that connects the most efficient strategies is called the efficiency frontier. Strategies that lie on the efficiency frontier are relevant for decision makers, whereas strategies below the line are considered dominated and inefficient.

Finally, incremental cost-effectiveness ratios of strategies on the efficiency frontier are compared to the relevant threshold. In Austria, there is currently no explicit cost-effectiveness threshold for the adoption of health 
technologies. Thresholds applied in other countries vary and are rarely cancer specific (Schwarzer et al. 2015). For example, in Canada the chair of the Ontario expert review committee suggested an oncology-specific ceiling threshold value of $\mathrm{C} \$ 75,000$ (this is equivalent to $\$$ US65,914 for 2009). NICE provided a general threshold in 2012 of $£ 18,317 /$ QALY (US $\$ 25,435$ ) that can be altered based on other factors.

\section{Sensitivity analyses}

We performed several one-way sensitivity analyses to account for parameter uncertainty ("second-order uncertainty"). This relates to the fact that the probabilities applied in the model are themselves uncertain: for example, values from clinical studies have confidence intervals. (Briggs et al. 2012a, b). The results are displayed in a modified tornado diagram that shows the variation in outcomes according to one parameter on a horizontal bar. Along the vertical axis, all relevant parameters are shown. The outcome of the base-case is indicated by a vertical line cutting through all horizontal bars. The longest bar reflects the most uncertain parameter (Briggs et al. 2012, b). We applied a content specific order of the parameters (bars), that is: age (40; 50 ; 70), discount rate $(0 ; 2.5 \% ; 5 \%)$, cost of chemotherapy $( \pm 10 \%)$, cost of ODX test set $( \pm 10 \%)$, utilities (95\% confidence intervals assuming beta distribution), distribution of AO risk groups $( \pm 20 \%)$ and probabilities of distant recurrence $(95 \%$ confidence interval, assuming beta distribution).

In addition, the results of our analysis from an Austrian perspective were compared to a Canadian cost-effectiveness study conducted by the Toronto Health Economics and Technology Assessment (THETA) Collaborative. (Paulden et al. 2013). The THETA group developed and applied a probabilistic Markov model (state-transition cohort model).
For our analysis, 100,000 patients were simulated per strategy, which was tested to provide stable results (Jahn et al. 2015).

\section{Results}

\section{Base-case analysis}

The results of the base-case analysis for Austria are displayed in Table 2. When compared to AO alone (NNN), using ODX in intermediate and high risk patients (NYY) increased discounted life years and QALYs by $2.8 \%$ (NNN: 14.33 LY, 11.08 QALY; NYY: 14.73 LY, 11.40 QALY). When using ODX in all patients (YYY) life years and QALYs increased by $4.1 \%$ (YYY: $14.92 \mathrm{LY}, 11.54$ QALY). However, costs also increased by $3.9 \%$ (NNN: 13,180 EUR, NYY: 13,710 EUR) to 13.9 \% (YYY: 15,930 EUR).

The base-case analysis shows that YYY (ODX provided to all patients) is the most effective strategy and is cost effective with an ICER of 15,700 EUR per QALY gained when compared to other technologies in health and medicine. Strategies NNN and NYY are also on the costeffectiveness frontier but they are less effective. Five of eight strategies were dominated (i.e., more costly and less effective: NNY, NYN, YNN, YNY, YYN).

In Table 3, further information on the benefits and harms of the evaluated strategies are displayed. Here we see that, for example, the strategy NYY leads to the fewest people $(0.0301 \%)$ suffering from adverse drug events (ADE). Providing ODX to all patients leads to a higher number of ADE $(0.0396 \%)$ compared to NYY but fewer patients develop recurrence (NYY: $0.2051 \%$; YYY $0.1722 \%$ ).

\section{Sensitivity analysis}

In the sensitivity analysis, different adjuvant chemotherapy treatment patterns were considered (Fig. 2). It was assumed that chemotherapy was provided to $13.73 \%$ of patients who had an intermediate risk according to $\mathrm{AO}$

\section{Table 2 Cost-effectiveness of ODX in the Austrian setting}

\begin{tabular}{|c|c|c|c|c|c|c|}
\hline \multirow[t]{2}{*}{ Strategy } & \multicolumn{4}{|c|}{ Base-case analysis } & \multirow[t]{2}{*}{$\Delta$ Total costs $\times 1000 €$} & \multirow[t]{2}{*}{$\overline{\text { ICER (€/QALY) }}$} \\
\hline & LYs & QALYs & $\Delta$ QALYs & Total costs $\times 1000 €$ & & \\
\hline 1-NNN & 14.33 & 11.08 & - & 13.18 & - & $N A^{a}$ \\
\hline 3-NYN & 14.46 & 11.18 & - & 13.36 & - & $D^{b}$ \\
\hline 2-NNY & 14.60 & 11.29 & - & 13.58 & - & D \\
\hline 4-NYY & 14.73 & 11.40 & 0.32 & 13.71 & 0.53 & 1628.35 \\
\hline 5-YNN & 14.46 & 11.17 & - & 15.48 & - & D \\
\hline 7-YYN & 14.64 & 11.31 & - & 15.61 & - & D \\
\hline 6-YNY & 14.76 & 11.41 & - & 15.81 & - & D \\
\hline 8-YYY & 14.92 & 11.54 & 0.14 & 15.93 & 2.22 & $15,727.78$ \\
\hline
\end{tabular}

a NA not applicable since NNN is the first comparator

b $\mathrm{D}$ dominated strategies discounted 
Table 3 Additional model outcomes

\begin{tabular}{llll}
\hline Strategy & $\begin{array}{l}\text { Adverse drug } \\
\text { event (\%) }\end{array}$ & $\begin{array}{l}\text { Number patients } \\
\text { with recurrence } \\
(\%)\end{array}$ & $\begin{array}{l}\text { Number of death } \\
\text { from recurrence }\end{array}$ \\
\hline 1-NNN & 0.0325 & 0.2677 & 26,078 \\
2-NNY & 0.0318 & 0.22828 & 22,186 \\
3-NYN & 0.0308 & 0.24287 & 23,636 \\
4-NYY & 0.0301 & 0.20509 & 19,863 \\
5-YNN & 0.0422 & 0.23507 & 22,905 \\
6-YNY & 0.0417 & 0.19817 & 19,290 \\
7-YYN & 0.0412 & 0.21193 & 20,593 \\
8-YYY & 0.0396 & 0.17215 & 16,705 \\
\hline
\end{tabular}

a Per 100,000 patients

and a low risk according to ODX in the base case (T1). In the sensitivity analysis that assumed none of $\mathrm{AO}$ intermediate/ODX low patients received chemotherapy (T2), the strategies NYY and YYY remained cost-effective with an ICER comparable to the base case. In addition, the strategy NYN (ICER: 501 EUR/QALY) was cost-effective (Additional file 1: Table S1).

Further sensitivity analyses found that strategies NYY and YYY remain on the cost-effectiveness frontier with thresholds below 5,300 EUR/QALY (NYY) and 47,000 EUR/QALY (YYY) (Tornado diagram Fig. 3; Additional file 2: Figure S1). Strategy YYY was dominated only when the probability of distant recurrence was increased (upper end of the $95 \%$ confidence interval). The strategy NYN was cost effective when we tested the lower and upper bound of the confidence interval for distant recurrence, $10 \%$ variation in costs, $20 \%$ variation in the $\mathrm{AO}$ risk group distribution, a lower discount rate $(2.5 \%)$ and younger patient population (40 years). The strategy NNY was cost effective when we tested the upper bound of the confidence interval for distant recurrence for patients who did not receive chemotherapy. All other test-treatment strategies remained dominated (Additional file 1: Table S1).

The comparison of the Austrian base-case analysis and the Canadian analysis by THETA is displayed in Fig. 4.

\section{Discussion}

We developed a decision-analytic model for the evaluation of the 21-gene assay in women diagnosed with ER and/or PR positive, HER-2/neu negative, and lymph node negative breast cancer in Austria. The model is flexible and can be adapted and applied to other countries and health care contexts.

We have demonstrated that the 21-gene assay is a costeffective tool for determining which ER/PR+, Her2/neu negative early breast cancer patients receive adjuvant chemotherapy when used in two scenarios in the Austrian context; first when used only in patients with a high or intermediate AO risk score (strategy NYY) (ICER

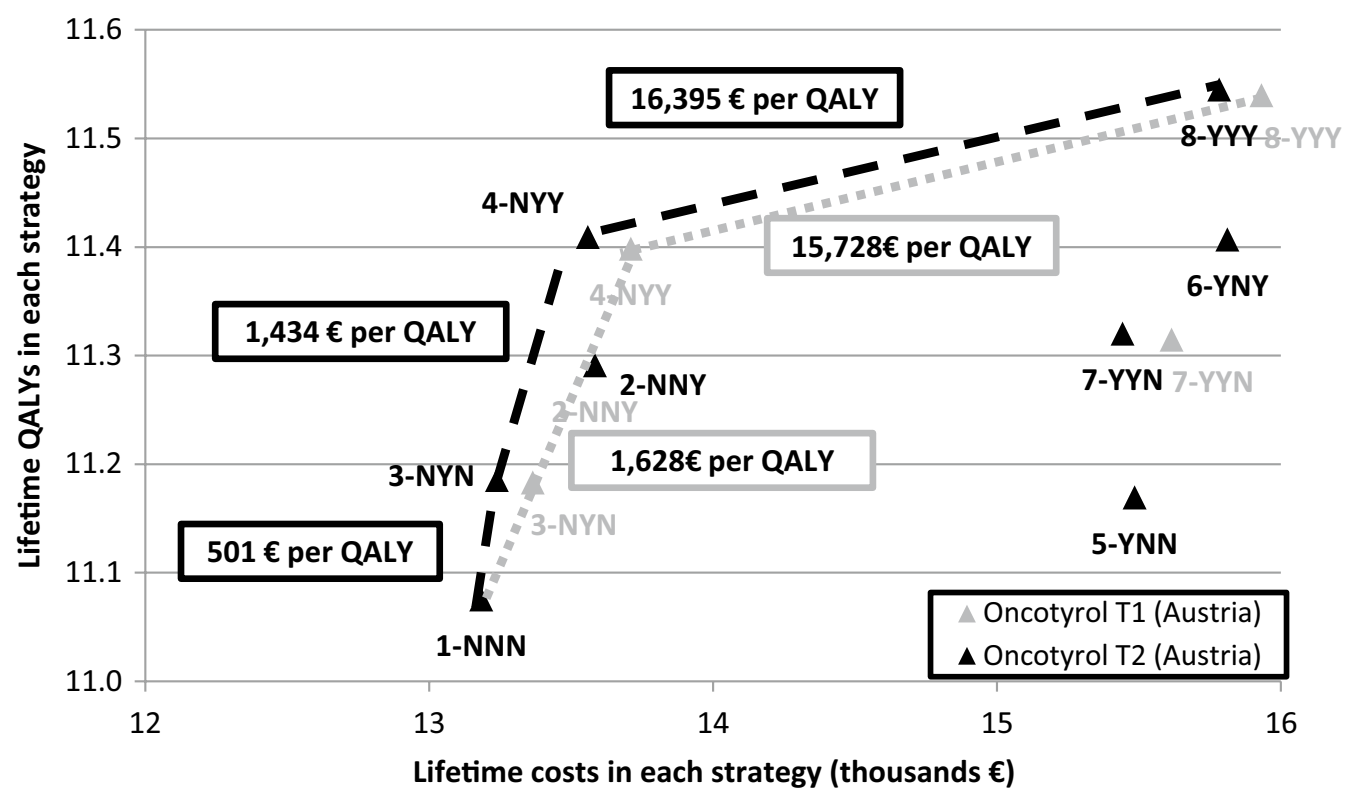

Fig. 2 Cost-effectiveness frontier for Austrian test-treatment strategy T1 (base case) and T2 (sensitivity analysis). Sensitivity analysis: risk group AO intermediate/ODX low receive no chemotherapy whereas in the base case $13.73 \%$ would receive chemotherapy, treatment for other risk groups as in base case); ICER - Incremental Cost-Effectiveness Ratios for the not dominated strategies that are on the frontier (ICER = additional costs/additional QALYs for the next most expensive strategy) 


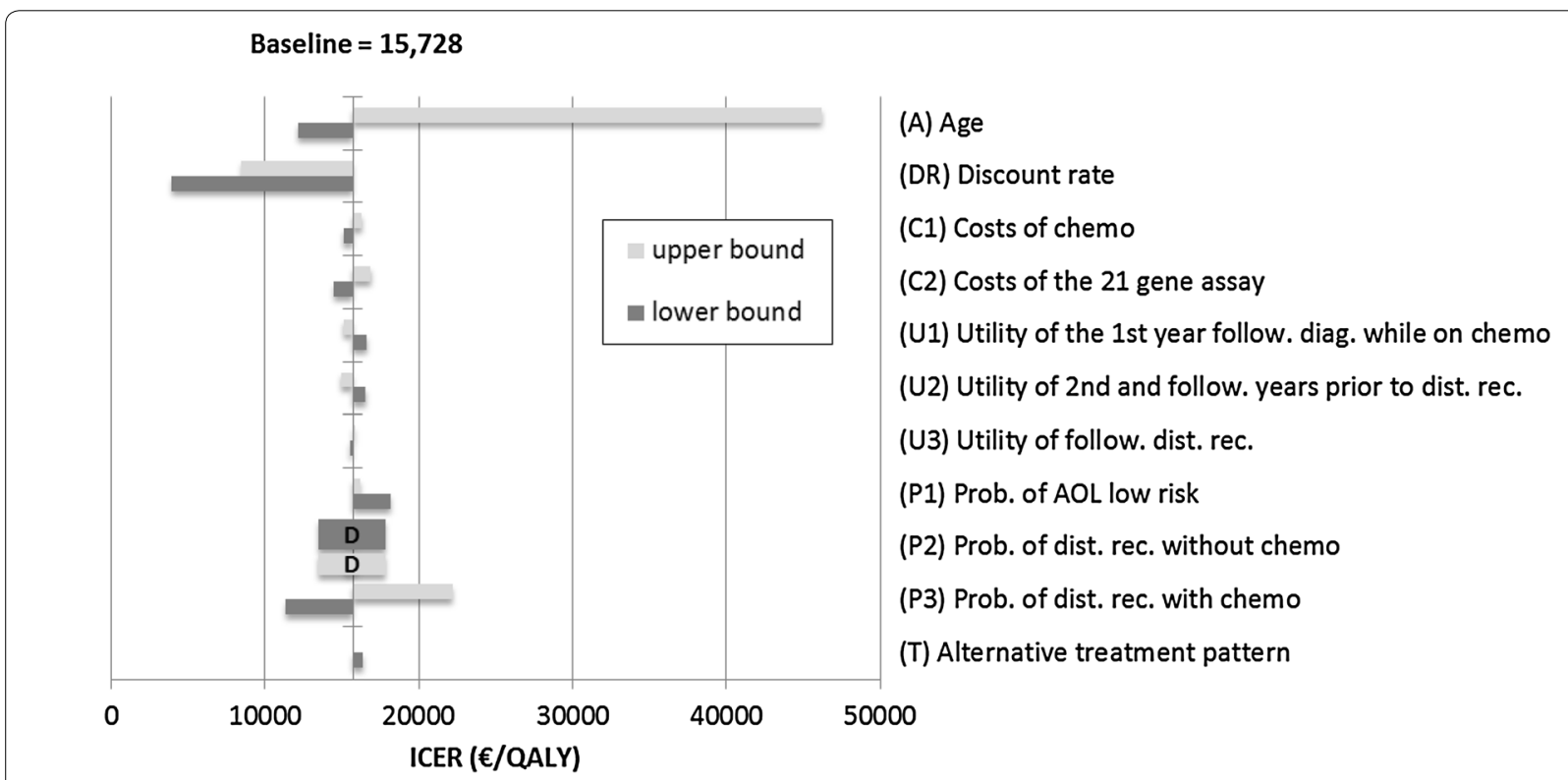

Fig. 3 Tornado diagram for the scenario YYY. prob. probability, dist. rec. distant recurrence, follow. following, diag. diagnosis; Parameter range: (A) $(40 ; 50 ; 70)(D R)(0 ; 2.5 \% ; 5 \%),(C 1)$ and (C2) $\pm 10 \%,(U 1)$ - (U3) $95 \%$ confidence intervals assuming beta distribution, (P1) $\pm 20 \%,(P 2)$ and (P3) $95 \%$ confidence interval assuming beta distribution, D dominated, AOL Adjuvant! Online

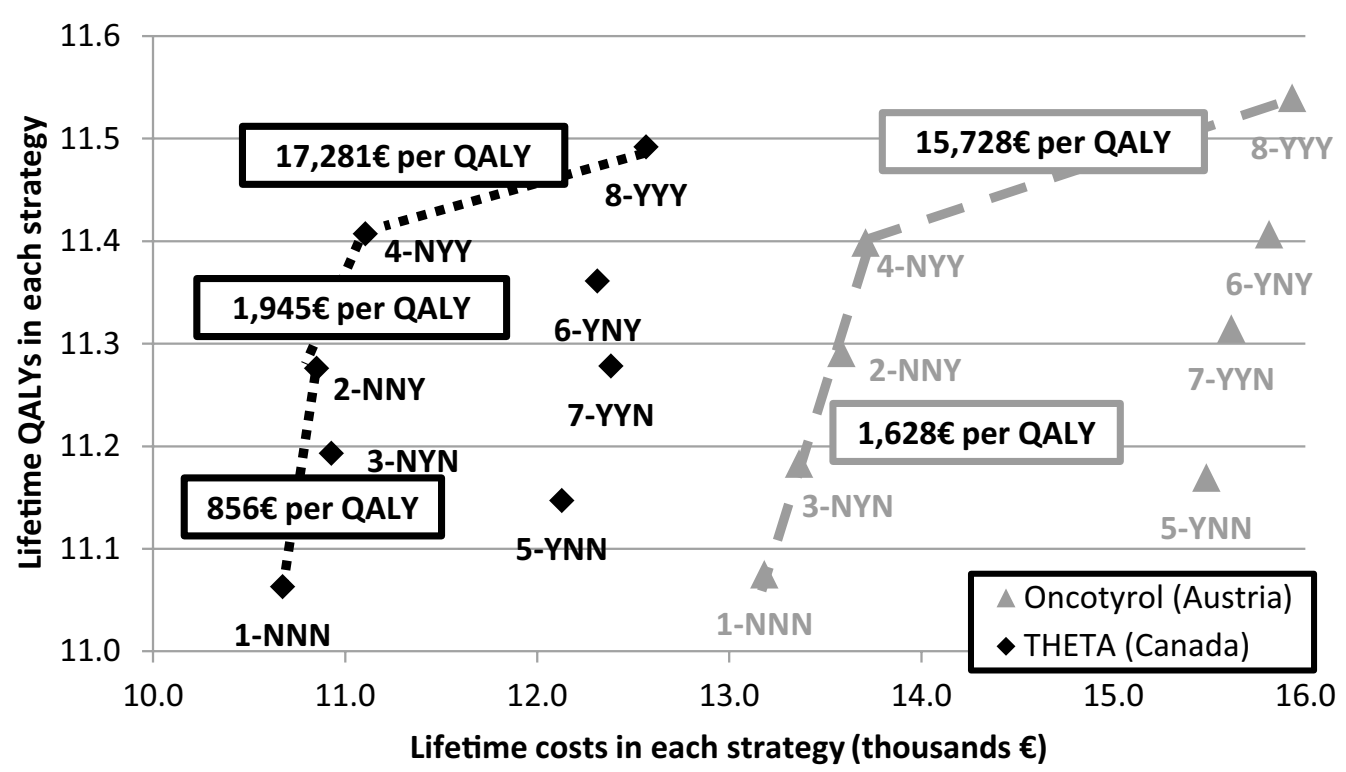

Fig. 4 Cost-effectiveness frontier comparison between Austrian and Canadian settings (incl. ICER values)

1600 EUR/QALY) and second, when used in all patients independent of the AO risk score (Strategy YYY) (ICER $15,700 \mathrm{EUR} / \mathrm{QALY})$. All other strategies that include testing of low risk AO (YNN, YNY, YYN) are dominated and should not be considered as cost-effective options. With respect to the sensitivity analysis, it seems that the strategy where only AO intermediate risk patients are tested (NYN) could also be a relevant option.
The modeling study shows that the 21-gene assay is cost effective for all AO risk groups. However, there is always the trade-off that the 21-gene assay uses resources that could be used elsewhere. Providing the genetic test to patients with $\mathrm{AO}$ intermediate or high risk only would be a cost-effective alternative that uses fewer total resources. However, drivers for the use of the 21-gene assay for $\mathrm{AO}$ low risk patients may include disagreements 
between physicians and patients, uncertainty and anxiety in patients as well as individual health considerations. The time for the genetic testing seems acceptable. The recurrence score is evaluated from a tissue sample sent to Genomic Health within 10-14 calendar days.

The 21-gene assay estimate can differ from the Adjuvant! recurrence estimate for several reasons. The 21-gene assay recurrence estimate is for distant recurrence only (risk of metastatic disease) while AO estimates the risk for all causes of recurrence (local, regional, contralateral breast cancer and distant recurrence). Because of this difference in the endpoint definition, Adjuvant!'s estimates of "risk of recurrence" are usually higher than those of the ODX test. However, the most appropriate comparisons are between the risk of breast cancer mortality as estimated by $\mathrm{AO}$ and the risk of distant recurrence as given by the ODX test, though an exact comparison is not possible (Sinn et al. 2013).

Several studies examined the cost effectiveness of ODX driven treatment (Klang et al. 2010; Kondo et al. 2008, 2011; Lyman et al. 2007; Paulden et al. 2013; Reed et al. 2009; Tsoi et al. 2010; Ward et al. 2013; Yang et al. 2012; Hornberger et al. 2005). In the majority of these studies, ODX was found to be superior to conventional risk classification methods (Klang et al. 2010; Kondo et al. 2008, 2011; Lyman et al. 2007; Tsoi et al. 2010; Hornberger et al. 2005). Hall et al. (2012) stated that ODX could be very cost effective for the NHS in the UK, but there is uncertainty in the evidence to support this claim. The Health Technology Assessment by Ward et al. (2013) reported that "compared with current clinical practice, OncotypeDX had a $12.4 \%$ (all women) and $91.6 \%$ (Nottingham Prognostic Index $>3.4$ ) probability of being considered cost effective when using a threshold of $£ 20,000$ per QALY gained respectively". Yang et al. (2012) compared two different gene expression profiles and concluded that Mammaprint to be even more effective and less costly than ODX. However, these analyses did not consider the provision of ODX conditional upon AO risk and therefore implicitly assumed that $\mathrm{AO}$ or conventional risk classification will be replaced rather than combined. Reed et al. (2009) cross-classified patients by clinicopathologic characteristics from AO and ODX. However, they assumed that in the absence of ODX test results, all patients with intermediate and high risk according to AO receive chemotherapy. They did not report test-treatment scenarios where only specific risk groups receive ODX. They estimated an ICER of USD10,788/QALY for an ODX guided therapy as compared to non ODX guided from a US societal perspective. With respect to the Canadian results of the THETA Collaborative, our findings are similar. They identified strategy NYY and YYY to be cost-effectiveness frontier with ICERs a little higher than those for Austria. In addition, in the Canadian setting, strategy NNY is cost effective. The cost-effectiveness frontier showed a similar shape with a slight shift right/up for Austria due to a little higher cost and higher QALYs. This can in part be the result of similar sources of utility data and probabilities of distant recurrence. However, countries differ with respect to chemotherapies that are provided. In addition, for Austria we did not consider end of life care and assumptions of chemotherapy toxicities differ. Hence, it was also found that the strategy NNY is cost effective in the analysis of the THETA collaborative but not for Austria.

Our model has several limitations. Though model parameters were carefully selected, some information was not available for Austria. Therefore, we adapted the distribution of the AO risk groups, utility parameters and estimates for the risk of distant recurrence from international studies (Lo et al. 2010; Paulden et al. 2013; Lidgren et al. 2007). Furthermore, since the 21-gene assay is not available in Austria, the treatment pattern was assumed based on the results of both risk scores as adapted from (Lo et al. 2010) with Austrian experts (Medical University Innsbruck 2012). In general, a review by Carlson and Roth (2013) concluded that there are a lack of studies reporting the impact of ODX on adjuvant chemotherapy use versus a standard approach. Individual health considerations and preferences may be more important in decisions about use of chemotherapy than the results of the 21-gene assay.

There is evidence that ODX results are associated with locoregional recurrence (Mamounas et al. 2010). Within our model, we did not consider the risk of local recurrence. Due to a lack of more detailed data, treatment of distant recurrence was assumed to be similar for all patients independent of the patient history. However, our microsimulation provides the flexibility to incorporate further personalized treatment decisions once that data become available.

ODX, as molecular signatures for ER-positive breast cancer show convincing analytical validity and clinical validity results. However, results have not yet proven robust clinical utility. In some cases, where this decision is difficult, ODX may be used in conjunction with all clinicopathological factors to inform treatment decisions (Azim et al. 2013; Senkus et al. 2013). However, future results from large phase III prospective clinical trials (TAILORx, RxPONDER) may confirm the accurate use of these new tools. Once further data are available, the model will be updated accordingly.

Further tests such as EndoPredict, Breast Cancer Index, MammaPrint, and Genomic Grade Index have shown promising results in ongoing trials. These tests differ slightly with respect to indication, whether they are 
prognostic or predictive, whether they must be conducted in a central lab, reference lab, or by local pathologists, technique (e.g., qRT-PCR, DNA micro array) outcomes and risk categories. Most tests require formalin-fixed, paraffin embedded tissue (Sinn et al. 2013). The EGAPP working group (EWG) launched by the CDC Office of Public Health Genomics in 2009 (EGAPP Working Group 2009) concluded that the evidence for the use of a gene expression profile is insufficient to assess the balance of benefits and harms for its proposed uses. The EWG encourages further evaluation of these technologies. Likewise, the IMPAKT 2012 Working Group suggested "a need to develop models that integrate clinicopathological factors along with clinical test" (Azim et al. 2013). In addition, Prat et al. (2012) created a dataset of 1380 patients to research the combination of PAM50, ODX, Mammaprint and SET. They showed that combinations of these signatures significantly increase prediction performance. Hence, for future cost-effectiveness studies, comparators and combinations must be thoroughly extended and selected.

Our model can support clinicians in their decision making as to whether the relatively small absolute benefit of chemotherapy in node-negative, ER-positive women outweighs the harm of adverse drug effects. Our discrete event simulation model produced useful results, and is available to be adapted for rapid assessment of new tests, variations in treatment strategies and additional biomarkers and treatments as they become available.

\section{Conclusion}

Our study suggests that ODX applied in all risk groups is the most effective strategy. Also the 21-gene assay is cost effective for patients independent of the AO risk score (Strategy YYY) (ICER 15,700 EUR/QALY) and in patients with a high or intermediate AO risk score (strategy NYY) (ICER 1600 EUR/QALY). Further research is needed to validate the 21-gene assay and use of AO in large prospective studies as well as real world registry studies. The results of future studies of other genetic tests may require alternative or additional test-treatment strategies.

\section{Additional files}

Additional file 1. Results one-way sensitivity analysis

Additional file 2. Tornado diagram for the scenario NYY

\section{Authors' contributions}

All authors have participated in the study to a sufficient extent to be named as authors. BJ, UR, CK, GS, MP, MH, US: contributed to the conception and design of the study, acquisition of data, analysis of data. BJ, UR, CK, GS, MH, MKR: contributed to the model development. BJ, UR, CK, MH, RM, GS, MP, MKL, MKR, US: contributed to the interpretation of the data, drafting and revising the article critically for important intellectual content, final approval of the version to be submitted. All authors read and approved the final manuscript.

\section{Author details}

${ }^{1}$ Institute of Public Health, Medical Decision Making and Health Technology Assessment, Department of Public Health, Health Services Research and Health Technology Assessment, UMIT - University for Health Sciences, Medical Informatics and Technology, Eduard Wallnoefer Center 1, A-6060 Hall i.T, Austria. ${ }^{2}$ Division of Public Health Decision Modelling, Health Technology Assessment and Health Economics, ONCOTYROL - Center for Personalized Cancer Medicine, Innsbruck, Austria. ${ }^{3}$ Department of Obstetrics and Gynaecology, Medical University Innsbruck, Innsbruck, Austria. ${ }^{4}$ Harvard Medical School, Beth Israel Deaconess Medical Center, Boston, MA, USA. ${ }^{5}$ Toronto Health Economics and Technology Assessment (THETA) Collaborative, University of Toronto, Toronto, ON, Canada. ${ }^{6}$ Department of Emergency Medicine, University of Alberta, Edmonton, AB, Canada. ${ }^{7}$ Center for Health Decision Science, Department of Health Policy and Management, T.H.Chan Harvard School of Public Health, Boston, MA, USA. ${ }^{8}$ Institute for Technology Assessment and Department of Radiology, Harvard Medical School, Massachusetts General Hospital, Boston, MA, USA.

\section{Acknowledgements}

We thank Michael Hofstetter (Institute of Public Health, Medical Decision Making and Health Technology Assessment, UMIT-University for Health Sciences, Medical Informatics and Technology, Hall i.T., Austria) for supporting the coding of the model.

\section{Competing interests}

Financial support for this study was provided in part by the COMET Center ONCOTYROL, which is funded by the Austrian Federal Ministries BMVIT/ BMWFJ (via FFG) and the Tiroler Zukunftsstiftung/Standortagentur Tirol (SAT). The funding agreement ensured the authors' independence in designing the study, interpreting the data, writing, and publishing the report. The following authors were/are employed by the sponsor: B. Jahn, U. Rochau, C. Kurzthaler, M. Kluibenschädl, G. Sroczynski, U. Siebert.

Received: 12 October 2015 Accepted: 14 October 2015

Published online: 01 December 2015

\section{References}

Adjuvant! Online Inc (2012) Decision making tools for health care professionals. http://www.adjuvantonline.com/index.jsp. Accessed 30 October 2012

Agency for Healthcare Research and Quality (2012) Breast cancer. http://www. ahrq.gov/browse/cancertypes.htm\#breast. Accessed 30 October 2012

Arbeitsgemeinschaft für gynäkologische Onkologie (AGO) der Österreichischen Gesellschaft für Gynäkologie und Geburtshilfe (OEGGG) (2012) Mammakarzinom. http://www.ago-manual.at/inhalt/imammakarzinom/12-risikofaktoren-vorsorge-und-genetik/. Accessed 9 August 2012

Asad J et al (2008) Does oncotype DX recurrence score affect the management of patients with early-stage breast cancer? Am J Surg 196(4):527-529

Azim HA Jr et al (2013) Utility of prognostic genomic tests in breast cancer practice: the IMPAKT 2012 Working Group Consensus Statement. Ann Oncol 24(3):647-654

Briggs AH et al (2012a) Model parameter estimation and uncertainty analysis: a report of the ISPOR-SMDM Modeling Good Research Practices Task Force Working Group-6. Med Decis Making 32(5):722-732

Briggs AH et al (2012b) Model parameter estimation and uncertainty: a report of the ISPOR-SMDM Modeling Good Research Practices Task Force-6. Value Health 15(6):835-842

Bryant J (2005) Toward a more rational selection of tailored adjuvant therapy. Data from the National Surgical Adjuvant Breast and Bowel Project. Presented at the primary therapy of early breast cancer. In: 9th international conference. St. Gallen, Switzerland

Cantor SB (1994) Cost-effectiveness analysis, extended dominance, and ethics: a quantitative assessment. Med Decis Making 14(3):259-265

Carlson JJ, Roth JA (2013) The impact of the Oncotype Dx breast cancer assay in clinical practice: a systematic review and meta-analysis. Breast Cancer Res Treat 141(1):13-22 
Caro JJ et al (2012) Modeling good research practices-overview: a report of the ISPOR-SMDM Modeling Good Research Practices Task Force-1. Med Decis Making 32(5):667-677

DGHO guidelines (2013) https://www.dgho.de/informationen/leitlinien. Accessed 22 Oct 2015

EGAPP Working Group (2009) Recommendations from the EGAPP Working Group: can tumor gene expression profiling improve outcomes in patients with breast cancer? Genetics in Medicine 11(1):66-73

Gnant M, Harbeck N, Thomssen C (2011) St. Gallen 2011: summary of the Consensus Discussion. Breast Care 2011(6):136-141

Gold MR, Siegel JE, Russell LB, Weinstein M (1996) Cost-effectiveness in health and medicine: report of the panel on cost-effectiveness in health and medicine. Oxford Univ Press, New York

Hall PS et al (2012) Economic evaluation of genomic test-directed chemotherapy for early-stage lymph node-positive breast cancer. J Natl Cancer Inst 104(1):56-66

Henry LR et al (2009) The influence of a gene expression profile on breast cancer decisions. J Surg Oncol 99(6):319-323

Hornberger J, Cosler LE, Lyman GH (2005) Economic analysis of targeting chemotherapy using a 21-gene RT-PCR assay in lymph-node-negative, estrogen-receptor-positive, early-stage breast cancer. Am J Manag Care 11(5):313-324

Hubalek M (2010) Mammakarzinom - Empfehlungen zu Diagnostik, Therapie und Nachsorgeuntersuchungen in Tirol. TAKO Vorstand, and und Mitwirkende. http://www.tako.or.at/files/Mamma_1_0.pdf. Accessed 30 October 2012

Jahn B et al (2012a) Cross-validation between discrete-event simulation model and a markov model_for personalized breast cancer treatment strategies [Oral Presentation]. In: 14th Biennial of the Society for Medical Decision Making (SMDM) European Meeting. Oslo, Norway

Jahn B et al (2012b) Cross-validation between discrete-event simulation model and a markov model_for personalized breast cancer treatment strategies. Value in Health 15(7):A647-A648

Jahn B et al (2015) Lessons learned from a cross-model validation between a discrete event simulation model and a cohort state-transition model for personalized breast cancer treatment. Med Decis Making [Epub ahead of print]

Karnon J et al (2012) Modeling Using Discrete Event Simulation: a Report of the ISPOR-SMDM Modeling Good Research Practices Task Force-4. Med Decis Making 32(5):701-711

Klang SH et al (2010) Economic implications of 21-gene breast cancer risk assay from the perspective of an Israeli-managed health-care organization. Value Health 13(4):381-387

Kondo $\mathrm{M}$ et al (2008) Economic evaluation of 21-gene reverse transcriptasepolymerase chain reaction assay in lymph-node-negative, estrogenreceptor-positive, early-stage breast cancer in Japan. Breast Cancer Res Treat 112(1):175-187

Kondo M et al (2011) Economic evaluation of the 21-gene signature (Oncotype DX) in lymph node-negative/positive, hormone receptorpositive early-stage breast cancer based on Japanese validation study (JBCRG-TR03). Breast Cancer Res Treat 127(3):739-749

Leitlinienprogramm Onkologie (OL) (2012) Interdisziplinäre S3-Leitlinie für die Diagnostik, Therapie und Nachsorge des Mammakarzinoms. http://www. krebsgesellschaft.de/download/S3_Brustkrebs_Update_2012_OL_Langversion.pdf. Accessed 30 October 2012

Liang $\mathrm{H}$ et al (2007) A retrospective analysis of the impact of oncotype DX low recurrence score results on treatment decisions in a single academic breast cancer center. In Genomic Health Abstract \#2061: Poster Session

Lidgren M et al (2007) Health related quality of life in different states of breast cancer. Qual Life Res 16(6):1073-1081

Lo SS et al (2010) Prospective multicenter study of the impact of the 21-gene recurrence score assay on medical oncologist and patient adjuvant breast cancer treatment selection. J Clin Oncol 28(10):1671-1676
Lyman GH et al (2007) Impact of a 21-gene RT-PCR assay on treatment decisions in early-stage breast cancer: an economic analysis based on prognostic and predictive validation studies. Cancer 109(6):1011-1018

Mamounas EP et al (2010) Association between the 21-gene recurrence score assay and risk of locoregional recurrence in node-negative, estrogen receptor-positive breast cancer: results from NSABP B-14 and NSABP B-20. J Clin Oncol 28(10):1677-1683

Medical University Innsbruck (2012) Expert Opinion, Medical Record Review, Innsbruck

Ontario Case Costing Initiative (2011) http://www.occp.com. Accessed 18 March 2013

Oratz R et al (2007) Impact of a commercial reference laboratory test recurrence score on decision making in early-stage breast cancer. Journal of Oncology practice 3(4):182-186

Oratz R et al (2011) Physician Survey of the Effect of the 21-Gene Recurrence Score Assay Results on Treatment Recommendations for Patients With Lymph Node-Positive, Estrogen Receptor-Positive Breast Cancer. J Oncol Pract 7(2):94-99

Paik S et al (2004) A multigene assay to predict recurrence of tamoxifentreated, node-negative breast cancer. N Engl J Med 351(27):2817-2826

Paik S et al (2006) Gene expression and benefit of chemotherapy in women with node-negative, estrogen receptor-positive breast cancer. J Clin Oncol 24(23):3726-3734

Paulden M et al (2013) Cost-effectiveness of gene expression profiling and adjuvant chemotherapy in women with early breast cancer. Value Health 16(5):729-739

Prat A et al (2012) Concordance among gene expression-based predictors for ER-positive breast cancer treated with adjuvant tamoxifen. Ann Oncol 23(11):2866-2873

Reed SD et al (2009) Cost effectiveness of ixabepilone plus capecitabine for metastatic breast cancer progressing after anthracycline and taxane treatment. J Clin Oncol 27(13):2185-2191

Schwarzer R et al (2015) Systematic overview of cost-effectiveness thresholds in ten countries across four continents. J Comp Eff Res 4(5):485-504

Senkus E et al (2013) Primary breast cancer: ESMO Clinical Practice Guidelines for diagnosis, treatment and follow-up. Ann Oncol 24(Suppl 6):vi7-vi23

Sinn P et al (2013) Multigene Assays for Classification, Prognosis, and Prediction in Breast Cancer: a Critical Review on the Background and Clinical Utility. Geburtshilfe Frauenheilkd 73(9):932-940

Statistik Austria (2012) G. Statistiken-Krebserkrankungen: Brust. http://www statistik.at/web_de/statistiken/gesundheit/krebserkrankungen/brust/ index.html. Accessed 30 October 2012

Statistik Austria (2012) G. Statistiken-Demographische Maßzahlen: Sterbetafeln. http://www.statistik.at/web_de/statistiken/bevoelkerung/ demographische_masszahlen/sterbetafeln/index.html. Accessed 12 December 2012

Tilak Financial Department, Cost Data Report (2012) Innsbruck (unpublished)

Tsoi DT et al (2010) Cost-effectiveness analysis of recurrence score-guided treatment using a 21 -gene assay in early breast cancer. Oncologist 15(5):457-465

Walter E (2012) IPF Institut für Pharmaökonomische Forschung, Report. Vienna (unpublished)

Walter E and Zehetmayr S (2006) Guidelines zur gesundheitsökonomischen Evaluation. Konsenspapier 156(23-24):628-632

Ward S et al (2013) Gene expression profiling and expanded immunohistochemistry tests to guide the use of adjuvant chemotherapy in breast cancer management: a systematic review and cost-effectiveness analysis. Health Technol Assess 17(44):1-302

Yang M, Rajan S, Issa AM (2012) Cost effectiveness of gene expression profiling for early stage breast cancer: a decision-analytic model. Cancer 118(20):5163-5170 